Gegensatz zur Mortalität von 100\% in den später als 24 Stunden nach Beginn operierten akuten Fällen ${ }^{1}$ ).

\begin{tabular}{|c|c|c|c|c|c|}
\hline & & $\begin{array}{l}\text { Operation } \\
\text { innerhalb }\end{array}$ & Pankreas & $\begin{array}{l}\text { Fettgewebs- } \\
\text { nekrosen }\end{array}$ & Ausgang \\
\hline I & 3 I a $0^{7}$ & I $2 \mathrm{~h}$ & \multirow{3}{*}{ ödematös } & $\theta$ & \multirow{3}{*}{ Heilung } \\
\hline 2 & $45 \mathrm{a} Q$ & $\mathrm{I} 2 \mathrm{~h}$ & & I & \\
\hline 3 & $40 a 0^{\pi}$ & $24 \mathrm{~h}$ & & + & \\
\hline 4 & $22 \mathrm{a} Q$ & $2 \times 24 \mathrm{~h}$ & \multirow{6}{*}{ nekrot.-hämorrh. } & $\theta$ & \multirow{6}{*}{$\uparrow$} \\
\hline 5 & $54 \mathrm{a} Q$ & $2 \times 24 h$ & & + & \\
\hline 6 & $46 \mathrm{a} \mathrm{O}^{\pi}$ & $2 \times 24 \mathrm{~h}$ & & + & \\
\hline 7 & $32 \mathrm{aO}$ & $3 \times 24 h$ & & + & \\
\hline 8 & $34 \mathrm{a} Q$ & $3 \times 24 \mathrm{~h}$ & & + & \\
\hline 9 & $46 \mathrm{a} ?$ & $4 \times 24 \mathrm{~h}$ & & + & \\
\hline Io & I9a $0^{\pi}$ & $\mathrm{I} 4 \times 24 \mathrm{~h}$ & nekrot.-seques triert & + & Heilung \\
\hline
\end{tabular}

Daraus folgt nahezu als Selbstverständlichkeit, daß die akute Pankreasnekrose innerhalb der ersten 24 Stunden operiert werden muß, wenn die Operation Aussicht auf Erfolg haben soll.

Wesentlicher sind aber die Schlußfolgerungen unter dem weiteren Gesichtspunkt des Zusammenhanges mit dem Gallensteinleiden. Während die ausgebildete akute Pankreasnekrose sich mehr als selbständiges Krankheitsbild darstellt mit Erscheinungen ähnlich einem Ileus, tragen die Fälle des Frühstadiums auch noch deutlich den Stempel einer Komplikation der Cholelithiasis. Nach vielleicht schweren und zahlreichen Anfällen in früherer Zeit setzt plötzlich ähnlich einer Perforation eine Attacke ein, die sich durch die unerhörte Stärke der Schmerzen und ihren Hauptsitz mehr nach links im Oberbauch von den früheren Anfällen unterscheidet. Die gewohnten Gallensteinschmerzen werden aber deutlich daneben noch gefühlt. Auch die Untersuchung ergibt noch klar die charakteristische Spannung und Druckempfindlichkeit in der Gallenblasengegend, daneben aber wieder auch Druckempfindlichkeit und Muskelspannung in der Mitte und weiter nach links im Oberbauch, wie sie gewöhnlichen Gallensteinanfällen fehlen.

Wenn also im Verlauf einer Cholelithiasis ein besonders heftiger Anfall einsetzt mit Schmerzen, die nach links in den Oberbauch ausstrahlen, mit Spannung oder auch nur Druckempfindlichkeit in dieser Gegend, so muß an die Möglichkeit einer beginnenden akuten Pankreasnekrose gedacht und dementsprechend sofort operiert werden.

Weiterhin beweisen aber diese bei der akuten Pankreasnekrose gemachten Beobachtungen ganz besonders einleuchtend die Zweckmäßigkeit einer Frühoperation des Gallensteinleidens, und zwar einer "Frühoperation" im doppelten Sinne.

Die Frühoperation im einzelnen Gallensteinanfall wird eine begleitende akute Pankreasnekrose im Beginn, eben im Stadium des Ödems treffen, sie verhindert die Ausbildung einer eigentlichen Nekrose mit allen ihren schweren Folgen.

Die Frühoperation im gewöhnlichen Sinne, d.h. also bezogen auf den ganzen Verlauf des Leidens, wird die Gallensteine noch in der Gallenblase fassen und mit ihr ausschalten, sie wird ihr Herabtreten in den Choledochus verhüten und damit die Hauptursache für die Komplikation einer Pankreasnekrose beseitigen.

Die alute Pankreasnekrose ist nur eine der zahlreichen drohenden Komplikationen des Gallensteinleidens, sie alle aber führen wieder zu dem einen Schluß: Zu der Forderung der technisch leichten, prognostisch günstigen Frühoperation.

Nachtrag: Vor kurzem konnte ich einen vierten gleichliegenden Fall beobachten, typisch in Anamnese und Befund: Ödem des Pankreas; mikroskopisch: entzündliche leichte In-

1) Einen vierten ganz typischen Fall dieser Art hat auch kürzlich Prof. SUDECK mit Prof. SCHOTTMULLLER zusammen beobachtet (demonstriert im ärztlichen Verein Hamburg): Patientin in den 30 er Jahren, früher Gallensteinanfälle, lange auf Magenleiden behandelt, jetzt plötzlich erkrankt unter perforationsähnlichen Erscheinungen im Oberbauch, operiert I2 Stunden nach Beginn des Anfalles. Odem scheinungen im Oberbauch, operiert I2 Stunden nach Beginn des Anfalles. Odem
des Pankreas und seiner Umgebung, keine Nekrosen des Drüsenparenchyms, keine des Pankreas und seiner Umgebung, keine Nekrosen des Drüsenparenchyms, keine Blutungen, nur Fettgewebsnekrosen in der Kapsel der Drüse. Steine in Gallen-
blase und Choledochus, in der Papilla Vateri ein Gallenstein fest eingeklemmt. Ausgang in Heilung! filtration ohne Schädigung des Parenchyms. Steine in aer geschrumpften Gallenblase. Choledochus nach Überwindung eines kleinen Hindernisses vor der Papille frei, im Stuhl dann Steine gefunden. In der Galle Bact. coli. Auffallende Erolung der vor der Operation fast pulslosen Frau, die sich jetzt auf dem Wege der Genesung befindet.

\section{DIE FUNKTIONSPRÜFUNG DER LEBER IN DER GRAVIDITÄT, ZUGLEICH EIN BEITRAG ZUR FRAGE DER RENALEN SCHWANGERSCHAFTS- GLYKOSURIE.}

\section{Von}

Dr. Géza Hetényi und Dr. Stefan Liebmann. Aus der III. med. Klinik (Direktor: Prof. Baron A. v. KoRÁNYI) und aus der Ii. Frauenklinik (Direktor: Prof. ST. v. TóTH) der Kgl, ung. Pázmány-Péter-Universität in Bưdapest.

Der wesentliche Einfluß der Schwangerschaft auf den Stoffwechsel des Organismus ist seit langem bekannt. Pathologischanatomische und histologische Befunde haben entschieden, daß auch die verschiedensten Organe eine Umwandlung im Laufe der Schwangerschaft erleiden. Daß sich auch die Funktion dieser Organe ändert, beweisen neuere Untersuchungen in erster Reihe betreffs der Drüsen mit innerer Sekretion.

Es liegt auf der Hand, daß jenes Organ, dessen Rolle in jedem einzelnen Teile des Stoffwechsels von hervorragender Wichtigkeit ist, welches sozusagen auch für den gesunden Organismus ein Stoffwechsel-Zentralorgan darstellt, - die Leber - ihre Funktion in der Schwangerschaft ebenfalls verändern wird. Diesbezüglich finden sich in der Literatur widersprechende Angaben, Einzelne fanden eine erhöhte Funktion der Leber, so SchirokaUter, der die Vermehrung der Leberdiastase in der Schwangerschaft registrieren konnte, während andere gar keine pathologische Veränderung der Leberfunktion nachzuweisen imstande waren. [BITTNER, Opitz, Schickele, Hein RichsdorfF auf Grund negativer anatomischer Leberbefunde, PFEIFFer, Schroeder, BARTels, Benere, Heynemann auf Grund des Nichtvorhandenseins der alimentären Lävulosurie bzw. der alimentären Galaktosurie (BAUCH).]

Größer ist jedoch die Zahl derjenigen, aus deren Untersuchungen auf eine Verminderung bzw. Insuffizienz der Leberfunktion geschlossen werden kann. So aus HofBauers path.-anat. Leberbefunden (Verfettung, Glykogenarmut, Gallenstauung), weiter aus den Ergebnissen CHvosteks, HoFBAUERS und der französischen Autoren die alimentäre Glykosurie, Kellers die Lävulosurie, ABDERHALdens und Fodors die Hyperaminosurie und erhöhte Polypeptidausscheidung betreffend, endlich aus der Arbeit CRAINCIANus' und Poppers bezüglich der Bilirubin- und Urobilinurie und des Verhaltens der hämoklastischen Krise.

Wie aus dieser kurzen Übersicht der Literatur ersichtlich, haben die meisten Autoren ihre Folgerungen aus dem Vorhandensein oder Fehlen eines einzigen Symptomes gezogen. Ja einzelne, hauptsächlich ältere Forscher basierten sogar auf solche Methoden, z. B. auf den positiven oder negativen Ausfall der alimentären Glykosurie, ihre Annahme, von denen heute allbekannt ist, daß sie mit den Veränderungen der Leberfunktion keinen gesetzmäßigen Zusammenhang zeigen und auch vom Zustande anderer Organe (Blutdrüsen, Nieren) in höchstem Maße beeinflußt werden.

Um zu entscheiden, in welcher Richtung sich die Leberfunktion Schwangerer verändert, erschien es uns zweckmäßig, Untersuchungen an größerem klinischen Material und mit Zuhilfenahme aller modernen funktionellen klinischen Untersuchungsmethoden anzustellen.

Nach dem Stande unseres heutigen Wissens können wir als solche Untersuchungsmethoden nur:

r. die Untersuchung der Urobilinogenausscheidung:

2. die alimentäre Hyperglykämie;

3. die alimentäre Lävulosurie

betrachten. 
Die Brauchbarkeit dieser Methoden wird heute allgemein anerkannt, dagegen hat uns die Untersuchung des Eiweißund Fettstoffwechsels bis heute noch keine geeignete Methode zur Beurteilung der Leberfunktion geliefert, sei es weil der größere Teil der Methoden nicht ganz spezifisch ist, sei es weil die sich brauchbar Erwiesenen eine zu komplizierte Methodik beanspruchen.

Das Material unserer Untersuchungen bildeten die im 7. bis 9. Monate seienden Hausschwangeren der II. Universitäts-Frauenklinik.

Nachfolgend unsere Ergebnisse:

I. Auf Urobilinogenurie untersuchten wir 43 Schwangere; von diesen zeigten 42 negative, eine jedoch schwache positive Alderhydreaktion. Letztere sahen wir bei einer 2I jährigen I. Grav. mens. VIII. In diesem Falle ergab die im Wochenbette wiederholte Untersuchung bereits ein negatives Resultat. - Diese verschwindend kleine $Z$ ahl der positiven Urobilinogenurie spricht jedenfalls gegen das allgemeine Vorhandensein einer verminderten Leberfunktion.

Von denen, die die Urobilinausscheidung gesunder Schwangeren studierten, kam LEMAIRE zu dem unserem gleichen negativem Ergebnisse; LIEPMANN läßt die Frage offen, wogegen MERLETTI auch bei gesunden Schwangeren eine Urobilinurie vorfand. Die Ausscheidung des Urobilins ist jedoch kein geeignetes Kriterium zur Annahme einer Leberläsion, da dieses als ein Kondensprodukt verschiedener, teilweise während des Stehens des Harnes sich bildender chemischer Substanzen $z u$ betrachten ist. Auch ist das Urobilin im normalen Harne regelmäßig nachzuweisen, und so kann in dieser Frage nur die Untersuchung der Urobilinogenausscheidung beweisend sein.

2. Auf alimentäre Hyperglykämie haben wir I 6 Schwangere untersucht. Im einzelnen Falle gingen wir folgendermaßen vor:

Nachdem wir uns überzeugten, daß keine spontane Zuckerausscheidung vorhanden ist, bestimmten wir zuerst den nüchternen Blutzuckerwert im Gesamtblut mit der BANGschen Mikromethode. Sodann bekamen die Schwangeren Ioo g Traubenzucker in Tee gelöst. 50 Minuten nachher neuerliche Blutzuckerbestimmung. Die Relation der $z$ wei Blutzuckerwerte ergibt, wie bekannt, den sog. hyperglykämischen Quotienten.

Die nüchternen Blutzuckerwerte schwankten zwischen $0,074-0,106 \%$, also binnen normalen Grenzen. Die alimentären Werte lagen zwischen $0,085-0,5_{5} \%$. In einem Falle betrug der alimentäre Blutzuckerwert - bei einem Nüchtern werte von $0, I_{4} \%-0,179 \%$. Der hyperglykämische Quotient befand sich zwischen den Werten von I,I2-I,47 und stieg bloB in dem obengenannten Falle auf 1,57. - Diese Blutzucker und Quotientenwerte, welch' letztere sich fast ausnahmslos unter $\mathrm{x}, 4$ befinden, schließen schon von vornherein mit größter Wahrscheinlichkeit das Vorhandensein einer Leberläsion aus, wie dies übrigens auch die an Leberkranken veranstalteten Untersuchungen unserer Klinik beweisen $^{1}$ ).

3. Die alimentäre Lävulosurie prüften wir in 25 Fällen. Nach Verabreichung von Ioo g Lävulose auf nüchternen Magen sahen wir in jedem einzelnen Falle positive Reaktion. Nach Ausschluß der spontanen Glykosurie wurde der Urin in stündlichen Intervallen durch vier Stunden untersucht.

Das häufige Vorkommen der alimentären Lävulosurie bei Schwangeren wurde von JÄGER, PFEIFFER (bei Eklamptischen $80 \%$ ), GugGisberg, Keller, MaNN, NeU-Keller beobachtet, während Schröder, Bartels, Beneke, HeyneMANN einen gegengesetzten Standpunkt vertreten.

Wenn wir die Ergebnisse unserer Untersuchungen zusammenfassen, scheint zwischen den, mit den zwei erstgenannten Methoden erhaltenen Ergebnissen und dem Ausfall der alimentären Lävulosurie ein scharfer Widerspruch $\mathrm{zu}$ bestehen. Während nämlich die äußerste Seltenheit der Urobilinogenurie und die niedrigen Werte des hyperglykämischen Quotienten entschieden gegen die Annahme einer verminderten Leberfunktion sprechen, würde die alimentäre

1) Siehe HETÉNYI, Deutsch. med. Wochenschr. I922.
Lävulosurie, welche uns von STrauss und UMBer als die beste Prüfungsmethode dargestellt wird, in schlagender Weise das Vorhandensein einer Leberläsion, respektive einer konsekntiven Hypofunktion in der Schwangerschaft, beweisen.

Nach den Erfahrungen aber, welche über das häufige Vorhandensein sowohl der alimentären wie auch der spontanen Glykosurie in der Schwangerschaft gemacht wurden, sahen wir uns gezwungen nachzuprüfen, ob das von uns gefundene allgemeine Vorkommen der alimentären Lävulosurie bei Schwangeren nicht auf dieselbe Ursache zurückgeführt werden kann, als die Häufigkeit der alimentären Glykosurie. Diese Ursache ist, wie dies die heutigen Autoren fast ohne Ausnahme anerkennen (Frank, Noorden, 'Guggisberg, Frank und Nothmann, JäGER usw.) in der gesteigerten Durchlässigkeit der Nieren zu suchen. Die Glykosurie wäre also eine renale. Von entscheidender Wichtigkeit waren in dieser Frage Blutzuckeruntersuchungen (JAKOBSEN, Frank, SCHIROKAUER, BÖNNIGER, RYSER), welche nachgewiesen haben, daß während der Blutzuckerwert nach alimentärer Zuckerzufuhr die normalen Grenzen nicht überschritt, Traubenzucker im Urin zumeist doch nachzuweisen war. Dies mußte auch bezüglich des Ursprunges der Lävulosurie entschieden werden.

Eine andere Frage, die durch den Vergleich der eigenen und der in der Literatur niedergelegten Ergebnisse entstanden ist war, wie das relativ häufigere Vorkommen der alimentären Lävulosurie der Glykosurie gegenüber zu erklären ist.

Zur Entscheidung der ersten Frage haben wir in I7 Fällen Blutzuckeruntersuchungen nach Einfuhr von roo g Lävulose angestellt. Die nüchternen Blutzuckerwerte bewegten sich auch in diesen Fällen zwischen den normalen Grenzen 0,076 bis $0,113 \%$. Die alimentären Blutzuckerwerte fanden wir zwischen $0,098-0,133 \%$, die Werte des hyperglykämischen Quotienten zwischen I,03-1,37. Diese Werte entsprechen vollkommen den bei lebergesunden nichtschwangeren Individuen gewonnenen Werten.

Im Sinne dieser Ergebnisse können wir im anhepatogenen (renalen) Ursprunge der alimentären Schwangerschaftslävulosurie nicht zweifeln.

Zur Klärung der zweiten Frage gingen wir denselben Weg, der bei ähnlichen Untersuchungen Nichtschwangerer befolgt wurde. Wir bestimmten nämlich in I7 Fällen, sowohl nach Einfuhr von Dextrose wie von Lävulose den hyperglykämischen Quotienten und beobachteten zur gleichen Zeit für beide Zuckerarten diejenige Höhe des Blutzuckerspiegels, bei welcher die Nieren den Zucker durchlassen. Wir sahen, daß die Nieren die Lävulose in I00\%, die Glykose aber nur in $59 \%$ (ro mal unter $\mathbf{I} 7$ Fällen) durchgelassen haben, obwohl der Blutzuckerwert bei keiner dieser Zuckerarten eine pathologische Höhe erreicht hat. Der Vergleich der Quotientenwerte ergab sodann, daß die Ausscheidung der Lävulose bereits bei einem niedrigen Blutzuckerspiegel beginnt als die gleicher Menge Glykose.

Auf Grund dieser Daten sehen wir uns veranlaßt, das häufige Vorkommen der Lävulosurie in der Schwangerschaft auf eine höhergradige Durchlässigkeit der Nieren für diese Zuckerart zurückführen zu müssen, was übrigens auch intravenöse Zuckerinfusionen bei Gesunden und Leberkranken bewiesen haben ${ }^{1}$.

Nachdem uns unsere Untersuchungen zur Annahme des anhepatogenen Ursprungs der alimentären Schwangerschaftslävulosurie berechtigen, verschwindet von selbst jener scheinbare Widerspruch, der zwischen den Ergebnissen der verschiedenen, von uns benützten Untersuchungsmethoden bestand. Wir können also behaupten, daß mit den klinischen Untersuchungsmethoden keine Verminderung der Leberfunktion in der Schwangerschaft nachauweisen ist.

$\mathrm{Ob}$ eine pathologische Veränderung der Leberfunktion in der entgegengesetzten Richtung, also erhöhte Tätigkeit anzunehmen ist, sollen weitere Untersuchungen entscheiden.

Nebenbei sei erwähnt, daß wir unsere Untersuchungen in 6 Fällen auch auf die alimentäre Galaktosurie (BAUER) aus- 
gedehnt haben. Nach Verabreichung von $20 \mathrm{~g}$ Galaktose auf nüchternen Magen bekamen wir viermal positive Reaktion im Harne. Schon der Umstand, daß auch bei Gesunden eine, im Vergleich zu anderen Zuckerarten geringere Menge Galaktose notwendig ist, um Zuckerausscheidung zu erzeugen, macht es unzweifelhaft, daß auch in diesen Fällen, also in der Schwangerschaft, der renale Komponent im Vordergrunde steht.

Endlich ermöglichten unsere Untersuchungen die Nachprüfungen von BERGSMAS Annahme, daß bei wiederholter Schwangerschaft, infolge der Dichtung des Nierenfilters, die alimentäre Zuckerausscheidung seltener wäre. Wir konnten keinen nennenswerten Unterschied zwischen Primi- und Multipara nachweisen. Bei der alimentären Glykosurie verteilten sich unsere positiven und negativen Fälle gleichmäßig. Bei der alimentären Lävulosurie waren alle Fälle positiv. Bei der alimentären Galaktosurie war die Zahl der positiv reagierenden Multipara sogar größer, als die der Primipara.

Zusammenfassung: $I$. Mit den heutigen, zur Funktionsprüfung der Leber geeigneten klinischen Untersuchungsmethoden (Urobilinogenurie, alimentäre Hyperglykämie) kann in der Schwangerschaft keine Verminderung der Leberfunktion nachgewiesen werden.

2. Die alimentäre Lävulosurie zeigte sich bei unseren Schwangeren in I0o\% positiv. - Obwohl diese Probe als Leberfunktions-Untersuchungsmethode allgemein anerkannt ist, ist sie $z u$ diesem $Z$ wecke in der Schwangerschaft - infolge der nachweisbar gesteigerten Durchlässigkeit der Nieren - unbrauchbar. Der Grund der alimentären Schwangerschaftslävulosurie ist eine anhepatogene (renale); welche Tatsache durch gleichzeitige Blutzuckeruntersuchungen exakt nachgewiesen werden kann.

3. Der Unterschied, der in der Niere für die Durchlässigkeit beider Zuckerarten auch unter physiologischen Verhältnissen besteht, zeigt sich auch in der Schwangerschaft. Dies erklärt die häufigere Nachweisbarkeit der alimentären Lävulosurie der alimentären Glykosurie gegenüber.

4. Die Prüfung der alimentären Zuckerausscheidung bei Multipara bietet keinen Anhaltspunkt für das Vorhandensein der von BerGSMA angenommenen Nierenfilterdichtung.

L iter a tur: LIEPMANN, Handb. d. Frauenheilk. Wiesbaden, I9I8, Bd. 3, S. I 54, I84, I86, 450. - Frank, Dtsch. med. Wochenschr. 1912, Nr. 6-7; Arch. f. exp. Pathol. u. Pharmakol. 72, 387; Zeitschr. f. phys. Chemie Nr. 70; Habilit.-Schr. Leipzig Vogel, I9I 3. - Frank u. Nothmann, Münch. med. Wochenschr. 1920, 50. Schirokauer, Berl. klin. Wochenschr. 1912, S. 500. - Bergsma, Zeitschr. f. Geburtsh. u. Gynäkol. 52. 19I2, - JäGER, Zeitschr. f. Geburtsh. u. Gynäkol. 74, 42-43. - ABDERIALdDEN u. Fodor, Zit. Aschner. - HofbaUer, Wien. klin. Wochenschr. I899, Nr. I; Zeitschr. f. Geburtsh. u. Gynäkol. 6I, 4I; Dtsch. med. Wochenschr. I9I3, 42; Arch. f. Gynäkol. 93. I9II; Volkmanns Samml. I910; Gynäkol. Io. - Pfeifferer, Dissert. Straßburg I9I3. - RySER, Dtsch. Arch. f. klin. Med. II8, H. 8. - GuGGISBERG, Gynäkol. Rundschau I917, H. I; 22. Monatsschr. f. Geburtsh. u. Gynäkol. 45, H. 4. - Noorden, Die Zuckerkrankheit, 7. Aufl. I9I7. JACOBSEN, Biochem. Zeitschr. 56. - BöNnIGER, Dtsch. med. Wochenschr. 1908, S. 780. - ManN, Zeitschr. f. klin. Med. 78, 488. Meyer-Betz, Erg. d. inn. Med. I2, 774. - Craincianu u. Popper, Zentralbl. (Kongreß) 18, 468. - HEINRICHSDORFF, Zeitschr. f. Geburtsh. u. Gynäkol. 70. 1912. 620. - BaUch, Monatsschr. f. Geburtsh. u. Gynälol. 42, 258. - OpItz, Zeitschr. f. Geburtsh. u. Gynäkol. 73, 35I. - NeU-Keller, Monatssçhr. f. Geburtsh. u. Gynäkol. I9I 3, H. 4. - Heynemann, Zeitschr. f. Geburtsh. u. Gynäkol. 7r. - Bitrner, Ref. Zentralbl. f. Gynälkol. 6, 488. Keller, Dissert. Heidelberg I9I3. - Chvoster, Wien. klin. Wochenschr. I909, H. 9. - SchrödER, Zeitschr. f. Geburtsh. u. Gynäkol. 56. - Bartels, Inaug.-Dissert. Halle, 1913. - Merdeti, Lemaire s. Meyer-Betz. - Schickele, Arch. f. Geburtsh. u. Gynäkol. Igio, Nr. 92. - Beneke, Zit v. Heynemann. - Strauss, Dtsch. med. Wochenschr. I90I, Nr. 44. - UmBer, Mohr Stähelins Handb. d. inn. Med. Bd. 3 .

Anm. b. d. Korrektur: In der Zwischenzeit erschien eine Arbeit von A. GOTTSCHALK in der $Z$. f. ges. exp. Med. $26, \mathrm{I}-2$., welche sich ebenfalls mit dem Funktionszustande der Leber in der Schwangerschaft beschäftigt. Zur Prüfung der Leberfunktion bediente er sich der von ISAAC angegebenen Methode der Verfolgung der Lävuloseglykämiekurve. Mit dieser Methode fand er unter 20 untersuchten Schwangeren sieben, bei denen der gesteigerte alimentäre Blutzuckerwert und die lane Dauter Leber schließen läßt. Auch häit er eine einmalige Blutzuckeruntersuchung nach der oralen Zuckerzufuhr für nicht genug stichhaltig. Bei Durchsicht seiner an- geführten Protokoile (Nr. I2-I8) fanden wir mit Ausnahme dreier Fälle (No. I2 $x 5$, x6) Ergebnisse, die den unseren durchaus entsprechen und unserer Meinung nach đie obere Grenze der normalen alimentären Hyperglykämie (Quotientenwert: I.4) nicht ibberschreiten. Ob demnach der Verlauf der Glykämiekurve allein als Zeichen einer verminderten Leberfunktion anzusehen ist, darüber haben wir vorläufig keine keine eigene Erfabrung. Nachiprüfungen werden dies entscheiden. Auf den Wer der Prïfung der Urobilinausscheidung haben wir bereits hingewiesen.

\section{ÜBER SCHWERE STÖRUNGEN DER DARM- MOTILITÄT ALS FERNSYMPTOM . EINER PROSTATITIS.}

\author{
Von \\ Dr. Oswald Schwarz, \\ Priv.-Doz. für Ürologie in Wien.
}

Es ist zur Genüge bekannt, daß die Prostatitis in allen ihren Stadien überaus polymorphe, vorwiegend in der sensiblen Sphäre lokalisierte Krankheitserscheinungen hervorrufen kann, die hinsichtlich ihrer klinischen Erscheinungsformen und ihrer möglichen Pathogenese auf den ersten Blick wenigstens jeden Zusammenhang mit der Prostata vermissen lassen. Man spricht dann von Reflex-, richtiger Fernsymptomen der Prostatitis, von denen Kreuzschmerzen, ischiasartige Schmerzen in beiden Oberschenkeln, Schmerzen in den Knien, Ureterkoliken imitierende Schmerzen im Unterbauch die geläufigsten sind (vgl. NotтнAfт).

In der letzten Zeit sah ich nun zufällig hintereinander einige Fälle, die ein untereinander fast völlig gleiches Krankheitsbild zeigten, in dem stürmische Symptome yon seiten des Darmes so sehr im Vordergrund standen, daß die $\mathrm{Pa}$ tienten bei der Wahl ihrer Ärzte und diese in ihrem diagnostisch-therapeutischen Vorgehen ausschließlich von diesen geleitet wurden. Es gelang mir jedoch, auch diese Symptome als Folgeerscheinungen einer Prostatitis zu enthüllen und erfolgreich zu behandeln.

Zum Arzt geführt wurden alle Patienten durch heftigste Krämpfe im rechten Unterbauch, die gegen die Mittellinie ausstrahlten und um den Nabel meist ein zweites Schmerzzentrum bildeten. Die Schmerzen traten mit größter Regelmäßigkeit in den späten Nachmittags- oder den ersten Morgenstunden (3-6 Uhr) auf; kam es endlich zum Abgehen von Flatus, so war der Anfall beendet. Immer bestand mehr oder weniger, einmal ein sehr hochgradiger Meteorismus, der zu Zwerchfellhochstand und Herzbeschwerden führte. Die Zustände wurden als akute Appendicitis, Darmspasmus, Darmatonie, einmal als Ileus gedeutet. Alle Untersuchungen, inklusive der Röntgendurchleuchtung ergaben bis auf einen Fall, in dem sich ein spastisch kontrahiertes Colon descendens fand, einen negativen Befund. Mit Rücksicht darauf und die Erfolglosigkeit aller medikamentös-diätetischen Therapie einigte man sich gewöhnlich auf die Diagnose Nierenkolik, weswegen mir die Fälle zur spezialistischen Untersuchung überwiesen wurden.

Ich fand nun bei allen Patienten in einem der beiden Prostatalappen ein haselnuß- bis kirschkerngroßes, weiches, äußerst druckempfindliches Infiltrat mit eitrigem Sekret, und behandelte den ersten Fall aus bestimmten Gründen, die weiteren schon unter der sicheren Annahme der ätiologischen Bedeutung dieser Infiltrate mit Prostatamassage. Gleich wach den ersten Massagen verloren die Anfälle bedeutend an Intensität und nach I4 Tagen, d. h. nach 6-8 Massagen, waren sie vollkommen verschwunden.

Einige Beispiele seien als Illustration des Gesagten angeführt: Der folgende Fall ist zwar nicht ganz typisch, dafür aber wegen einiger Details von Interesse, er war zugleich der erste, der mich auf den hier behaupteten Zusammenhang zwischen Prostatitis und den. Darmsymptomen führte.

Fall $I$. Ein 50 jähriger, beleibter Herr erkrankte plötzlich an heftigsten Koliken in der linken Unterbauchgegend, die in den Hodensack ausstrahlten und mit Anschwellung des linken Hodens einhergingen; gleichzeitig bestand hochgradiger Meteorismus. Der Pat. hatte von einer 8 Jahre zurückliegenden Hernienoperation 3 Finger oberhalb dem linken Poupartschen Bande eine etwas dehiszente Narbe und diese wurde von mehreren konsultierten 